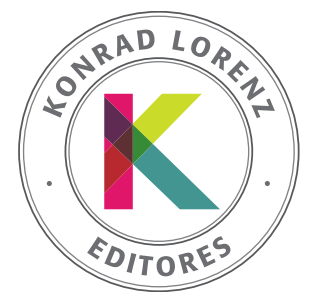

http://sumapsicologica.konradlorenz.edu.co

\title{
Evidências preliminares de validade de versão reduzida da escala de compra por impulso de Rook e Fisher
}

\author{
Tiago Azevedo Marot ${ }^{a, *}$, Samuel Lincoln Bezerra Lins ${ }^{b}$
}

\author{
a Departamento de Psicologia, Pontifícia Universidade Católica do Rio de Janeiro, Brasil \\ ${ }^{b}$ Departamento de Psicologia, Universidade do Porto, Porto, Portugal
}

Recebido em 8 de março de 2021; aceito em 25 de maio de 2021

\author{
PALAVRAS-CHAVE \\ Compra por impulso, \\ versão reduzida, análise \\ fatorial confirmatória \\ multigrupo, endividamento, \\ materialismo
}

Resumo Introdução: A redução de escalas visa a redução da quantidade de itens, mantendo-se suas qualidades psicométricas. 0 presente estudo teve como objetivo a redução da versão brasileira da escala de compra por impulso de Rook e Fisher. Método: Participaram do estudo 1173 brasileiros, sendo 868 mulheres e 305 homens com a média de idade de 24.8 anos e desvio-padrão de 12.7. Todos os participantes foram contatados via redes sociais ou e-mail. A pesquisa foi dividida em três estudos. Resultados: No primeiro estudo, a estrutura unifatorial do instrumento foi corroborada, uma vez que a análise paralela sugeriu a retenção de um fator e os índices de ajuste foram acima de .95. Foram selecionados quatro itens para comporem a versão reduzida. 0 segundo estudo analisou a estrutura interna e as cargas fatoriais da escala reduzida por meio da análise fatorial exploratória e da análise fatorial confirmatória. Os resultados da análise fatorial exploratória foram semelhantes aos do estudo 1 e a análise fatorial confirmatória também apresentou índices de ajuste acima de .90 . No terceiro estudo foram realizadas correlações para verificar o grau de diferença das correlações entre a escala reduzida e a original. Foram obtidas evidências de validade preditiva com as escalas de materialismo, consumo de status e propensão ao endividamento. Ainda, realizou-se uma análise fatorial confirmatória multigrupo que verificou a invariância do instrumento entre homens e mulheres. Conclusão: Conclui-se que a escala reduzida de compra por impulso, 4- $\mathrm{Cl}$, é considerada adequada para a mensuração desse construto.

( 2021 Fundación Universitaria Konrad Lorenz. Este é um artigo Open Access distribuído sob os termos da Licença Creative Commons CC BY-NC-ND (http://creativecommons.org/licenses/ by-nc-nd/4.0/).

\footnotetext{
* Autor para correspondência.

Correio eletrônico: marottiago@gmail.com
} 


\section{KEYWORDS:}

Impulsive buying, brief version, multigroup confirmatory factor analysis, indebtedness, materialism

\section{Preliminary Evidence of Validity of the Reduced Version of Rook and Fisher's Impulse Buying Scale}

Abstract Introduction: Scale reduction aims the reduction of the number of the items maintai-
ning its psychometric properties. The goal of this study was to reduce the Brazilian version of
Rook and Fisher's impulsive buying scale. Method: Participants were 1173 Brazilians, in which
868 were women and 305 were men with mean of age of 24.8 years old and a standard deviation
of 12.7. All participants were recruited via social media or e-mail. The research was split in three.
Results: In the first study, the unifactorial structure was supported, once the parallel analysis
suggested the retention of one factor, and the fit indexes were superior to .95. Four items were
selected to form the reduced version. The second study analyzed the internal structure and the
factorial loadings of the reduced scale through exploratory factor analysis and confirmatory fac-
tor analysis. The results from the exploratory factor analysis were similar to those of study 1 and
the confirmatory factor analysis also showed fit indexes above .90. In the third study, correlation
analysis were run to verify the disparities between the reduced and the original scale. Predictive
validity evidence was gathered with the materialism, consumption by status, and propension to
indebtedness. Still, a multigroup confirmatory factor analysis verified the instrument invariance
between men and women. Conclusion: It is concluded that the reduced impulsive buying scale,
4-CI, is considered adequate to the measurement of this construct.
(c) 2021 Fundación Universitaria Konrad Lorenz. This is an open access article under the CC BY-NC-ND
license (http://creativecommons.org/licenses/by-nc-nd/4.0/).

\section{Compra por impulso}

A compra por impulso é um dos vários tipos de comportamento de compra. Esse comportamento diz respeito à baixa ponderação cognitiva e à alta busca por prazer imediato através da obtenção de novos produtos (Rook \& Fisher, 1995). Como forma de incentivar este comportamento de compra, destacam-se as ações do mercado de frequentes lançamentos de novos produtos a preços reduzidos (e.g. fast-fashion), levando os consumidores a vivenciar altos níveis de excitação, resultando na compra impulsiva (Xiao \& Nicholson, 2011). Devido a esse novo padrão de vendas de produtos, a compra por impulso tornou-se ainda mais evidente, uma vez que ela é potencializada por estratégias de venda (Cook \& Yurchisin, 2017).

Dessa forma, diversos estudos no campo da psicologia têm estudado o comportamento de consumo (Farah \& Ramadan, 2020; Fenton-O’Creevy et al., 2018; Lim et al., 2020). Dentre esses estudos, a compra por impulso ganhou evidência como um construto muito estudado, não só na área da psicologia, mas também no marketing e em outras áreas afins (Ahn et al., 2020; Efendi et al., 2019). No Brasil, por exemplo, a influência de descontos (Santini et al., 2014) e de diferentes estratégias de marketing (Dias et al., 2014) sobre compra por impulso são exemplos de estudos experimentais.

Diferentes definições de compra por impulso são conhecidas no meio científico e, consequentemente, diversas formas de sua mensuração também são. Das definições de compra impulsiva, a proposta por Stern (1962) se destaca por ser a pioneira nesse campo. Stern (1962) divide a compra por impulso em quatro tipos: (1) a compra por impulso pura, (2) a compra por impulso lembrada, (3) a compra por impulso de sugestão e a (4) compra por impulso planejada. Foi a partir das pesquisas de Stern (1962) que as atuais definições de compra por impulso se originaram. A definição mais conhecida é a estabelecida por Rook (1987), que define a compra por impulso como uma urgência poderosa e persistente de comprar imediatamente. Alguns anos mais tarde, Rook e Fisher (1995) constroem uma escala unidimensional que avalia a compra por impulso, definindo-a como a tendência da pessoa a "comprar de forma espontânea, sem reflexão, imediata e atraída emocionalmente pela gratificação instantânea" (Rook \& Fisher, 1995, p. 306).

Outras escalas que propõem a mensuração da compra por impulso são a Tendência de Compra por Impulso, criada por Beatty e Ferrel (1998) e a Escala de Compra Impulsiva, elaborada por Puri (1996). Vale ressaltar que a última tem o objetivo de aferir a impulsividade do consumidor, sendo uma escala composta por dois fatores cognitivos: Prudente e Hedônico. Na mesma linha de Puri (1996), Verplanken e Herebadi (2001) e Youn e Faber (2002), desenvolveram também escalas com duas dimensões, sendo elas a Cognitiva e a Afetiva. Ainda, atenta-se para estudos como os de Youn e Faber (2002) e Sharma et al. (2011) em que, além de considerar os fatores afetivos e cognitivos, eles adicionaram um fator comportamental. Eles verificaram, entretanto, que o fator comportamental se expressava apenas em culturas consideradas coletivistas, enquanto nas culturas individualistas, esse novo fator se aglutinava com a dimensão afetivo.

No presente estudo, a escala trabalhada foi a de Padilha et al. (2015). Essa escala é a versão brasileira da escala de compra por impulso de Rook e Fisher (1995). A escala inicialmente construída é composta por nove itens com cargas fatoriais que variam de .60 a .81 e valor alfa de .88 (Rook \& Fisher, 1995). Estudos relacionados à compra por impulso têm sido realizados em diferentes contextos (e.g., Vietnã, Turquia, Suécia, Reino Unido, Paquistão, etc.) demonstrando adequados índices psicométricos dessa escala (Ahmad et al., 2019; Cakanlar \& Nguyen, 2019; Spiteri Cornish, 2020). No Brasil, Padilha et al., (2015) realizaram um estudo de adaptação da escala que apresentou alfa de Cronbach adequado $(\alpha=.87$ ) com $51.2 \%$ de variância explicada e, posteriormente, Aquino et al. (2020) apresentaram novas evidências de validade para o mesmo instrumento. 


\section{Compra por impulso e relação com outras variáveis}

Além da compra por impulso, outras variáveis relacionadas às compras também foram estudadas pela comunidade científica. Construtos como materialismo (Mette et al., 2019), endividamento (Vieira et al., 2014), consumo por status (Eastman et al., 1999; Strehlau \& Aranha, 2004), prazer nas compras (Santos \& Fernandes, 2006) e consumo conspícuo (Chung \& Fischer, 2001) são alguns exemplos. Em vista disso, foram incluídas na presente pesquisa as escalas de materialismo, consumo por status e propensão ao endividamento para que suas relações da escala original e a escala reduzida fossem comparadas.

Os estudos de Tatzel (2002) e Watson (2003), por exemplo, sugeriram a existência de relações diretas entre os níveis de materialismo e os de compra por impulso, em que as pessoas com maiores níveis de materialismo tendiam a ter atitudes mais positivas frente à compra por impulso e a gastar mais nas compras. Isso se dá porque o materialismo é compreendido como um fenômeno em que a pessoa entende a aquisição de bens materiais como fator central para a felicidade e o sucesso na vida (Richins \& Dawson, 1992) e que, portanto, reforça a relação com a compra por impulso (Podoshen \& Andrzejewski, 2012).

Em relação ao consumo por status, Eastman et al. (2013) encontraram em seu estudo relações positivas entre os níveis de compra por impulso e o consumo por status. Eles indicam que essa relação se dá devido à necessidade das pessoas de exibir seus status por meio de bens materiais (consumo por status) e que resultaria na compra impulsiva de produtos que elevariam seu prestígio social.

Por fim, a compreensão da relação entre a compra por impulso e propensão ao endividamento é dada pela influência que a compra impulsiva teria sobre a tendência de contração de dívidas devido à ausência de organização de compras. Omar et al. (2014) encontraram uma correlação positiva entre a compra por impulso e a contração de dívidas, indicando a relação existente entre essas duas variáveis.

\section{Escalas reduzidas}

Escalas reduzidas têm se tornado populares em Psicologia uma vez que os instrumentos são respondidos mais rapidamente, os participantes são menos suscetíveis à fadiga e torna-se menos problemático o uso de um maior número de escalas (Gosling et al., 2003). Ainda, ressalta-se que instrumentos mais breves facilitam estudos transculturais devido à sua maior estabilidade dos índices de fidedignidade e de sua estrutura fatorial (Thompson, 2007). De Holanda Coelho et al. (2018), por exemplo, realizaram o refinamento da escala de necessidade de cognição (Cacioppo \& Petty, 1982) e suportaram a hipótese de que os instrumentos reduzidos apresentam índices psicométricos semelhantes aos da escala original.

\section{Visão geral}

Em vista da relevância do desenvolvimento de uma escala reduzida que mensure a compra por impulso, o presente artigo teve como objetivo reduzir a escala de compra por impulso de Padilha et al. (2015) e avaliar suas características psicométricas. Para tanto, foram realizados três estudos.
O primeiro estudo avaliou a estrutura fatorial do instrumento e permitiu a escolha de quatro itens para comporem a versão reduzida. No segundo, foram analisadas a estrutura interna e cargas fatoriais por meio da análise fatorial exploratória e de análise paralela em uma subamostra, e em uma segunda subamostra foi realizada uma análise fatorial confirmatória. No último estudo foram realizadas correlaçõos e uma análise fatorial confirmatória multigrupo.

\section{Estudo 1}

O objetivo do primeiro estudo foi avaliar a estrutura fatorial da escala de compra por impulso a fim de selecionar os potenciais itens para a escala reduzida.

\section{Método}

\section{Participantes}

Participaram do estudo 1.581 adultos brasileiros, com a média de idade de 24.6 anos $(D P=12.9)$, sendo $76 \%$ mulheres e $24 \%$ homens. Em relação ao grau de formação dos respondentes, $86.9 \%$ possuíam, pelo menos, o ensino superior completo, $10.5 \%$ possuíam ensino médio completo e $2.6 \%$ ensino fundamental completo. A maior parte da amostra foi composta de participantes da região Sudeste do Brasil (51.7\%), seguido do Nordeste (18.9\%), Sul (17.3\%), Centro-Oeste $(9.8 \%)$ e Norte, $2.3 \%$.

\section{Instrumentos}

Escala de Compra por Impulso, versão brasileira (Padilha et al., 2015). Essa escala é unidimensional e é composta por nove itens (e.g., "Eu costumo comprar coisas sem pensar") respondidos em formato Likert de sete pontos, sendo 1 = discordo totalmente e 7 = concordo totalmente. Quanto maiores os escores nessa escala, maior a propensão à comportamentos de compra por impulso.

\section{Procedimentos}

De coleta. Os participantes foram recrutados por meio de convites disponibilizados por e-mails e redes sociais. Os participantes somente poderiam responder o questionário após declararem aceitar o Termo de Consentimento Livre e Esclarecido. 0 estudo foi aprovado pelo comitê de ética em pesquisa com seres humanos da Pontifícia Universidade Católica do Rio de Janeiro (PUC-Rio).

De análise. Foi realizada uma análise fatorial exploratória (AFE) no Software FACTOR (Ferrando \& Lorenzo-Seva, 2017) para verificar a unidimensionalidade do instrumento e as cargas fatoriais dos itens. 0 teste de esfericidade de Bartlett e o procedimento de adequação da amostra Kaiser-Meyer-Olkin (KMO) foram realizados para verificar o cumprimento dos pré-requisitos da escala para a realização da AFE. O método de extração de fatores utilizado foi o Robust Diagonally Weighted Least Squares (Asparouhov \& Muthén, 2010). 0 critério para a extração de fatores foi o critério de análise paralela (Timmerman \& Lorenzo-Seva, 2011). O índice de confiabilidade utilizado foi o McDonald's Ômega (McDonald, 1999). Para verificar a adequação do modelo, foram utilizados os índices Root Mean Square Error of Aproximation (RMSEA), CFI e TLI. Os valores de RMSEA devem ser menores que .80 e de CFI e TLI acima de .90 (Brown, 2006). 


\section{Resultados}

Os resultados sugeriram que a escala é fatorável (Teste de esfericidade de Bartlett $=2303.2$, $g l=36 ; p<.001$; $K M O=.91)$. $O$ método de extração de fatores apontou para uma escala unifatorial como sendo a mais adequada, explicando 77.2 \% da variância total dos dados (Tabela 1), com o valor do coeficiente Ômega de .91. 0 mesmo resultado também foi encontrado pela técnica da Análise Paralela, com permutação aleatória dos dados observados (Horn, 1965; Lorenzo-Seva \& Ferrando, 2013; Timmerman \& Lorenzo-Seva, 2011), uma vez que apenas um fator oriundo dos dados reais obteve variância explicada maior que fatores aleatórios (Variância dos dados reais, Fator 1: 79.56\%, Fator 2: $10.10 \%$; Variância dos dados aleatórios, Fator 1: $35.11 \%$, Fator 2: $27.26 \%)$. Os índices de ajuste e de resíduo se demonstraram satisfatórios: $\chi^{2}=92.8 ; \mathrm{gl}=27 ; p<.001 ;$ RMSEA $[95 \% \mathrm{IC}]=.065[.049-.076] ; C F I=.992 ; T L I=.989$.

Para a redução do instrumento, os quatro itens com maiores cargas fatoriais foram selecionados para verificar tanto a estrutura fatorial e a variância explicada em uma nova amostra.

Tabela 1. Análise fatorial exploratória da escala de compra por impulso $(n=581)$

\begin{tabular}{|c|c|}
\hline Item & Carga fatorial \\
\hline 3. Eu costumo comprar coisas sem pensar & .88 \\
\hline $\begin{array}{l}\text { 2. "Simplesmente compro"; isto descreve a } \\
\text { maneira como eu compro as coisas }\end{array}$ & .82 \\
\hline $\begin{array}{l}\text { 4. "Eu vejo, eu compro"; esta afirmação } \\
\text { me descreve }\end{array}$ & .80 \\
\hline $\begin{array}{l}\text { 6. "Compro agora e penso sobre isto mais } \\
\text { tarde"; esta afirmação me descreve }\end{array}$ & .76 \\
\hline $\begin{array}{l}\text { 7. Eu compro as coisas de acordo como me } \\
\text { sinto no momento }\end{array}$ & .61 \\
\hline $\begin{array}{l}\text { 1. Eu frequentemente compro coisas es- } \\
\text { pontaneamente }\end{array}$ & .56 \\
\hline $\begin{array}{l}\text { 8. Eu planejo cuidadosamente a maioria de } \\
\text { minhas compras (invertido) }\end{array}$ & .54 \\
\hline $\begin{array}{l}\text { 5. Às vezes eu fico com vontade de com- } \\
\text { prar coisas no impulso do momento }\end{array}$ & .53 \\
\hline $\begin{array}{l}\text { 9. Às vezes eu sou um pouco distraído } \\
\text { sobre o que eu compro }\end{array}$ & .43 \\
\hline Eingenvalue & 5.19 \\
\hline Variância total explicada & 77.2 \\
\hline Média $(D P)$ & $3.24(1.09)$ \\
\hline Coeficiente Ômega & .91 \\
\hline
\end{tabular}

\section{Estudo 2}

O segundo estudo buscou verificar as propriedades psicométricas da escala de compra por impulso reduzida (4$\mathrm{Cl}$ ), bem como avaliar sua estrutura interna. Dessa forma, os resultados obtidos derivaram de duas subamostras. A primeira para a realização da análise fatorial exploratória, e a segunda para a análise fatorial confirmatória.
Método

\section{Subamostra 1}

\section{Participantes}

A primeira amostra foi composta por 230 adultos brasileiros, sendo $73 \%$ da amostra composta mulheres e $27 \%$ homens, a média de idade foi de 25.2 anos ( $D P=12.4$ ), 88.3\% possuíam, ao menos, o ensino superior completo, 9.1\% possuíam Ensino Médio completo e 2.6\% Ensino Fundamental completo. A maior parte da amostra foi composta de participantes da região Sudeste do Brasil (49.1\%), seguido do Nordeste (22.2\%), Sul (18.2\%), Centro-Oeste (6.1\%) e Norte, (4.4\%).

\section{Instrumentos}

Escala de compra por impulso reduzida de 4 itens $(4-\mathrm{Cl})$.

\section{Procedimentos}

De coleta. Para a coleta de dados, foram empregadas as mesmas técnicas de coleta mencionadas no Estudo 1.

De análise. Os mesmos procedimentos empregados no Estudo 1 foram adotados na primeira amostra do Estudo 2 $(n=230)$. Foi realizada uma nova análise fatorial exploratória (AFE) para que os resultados das amostras fossem comparados.

\section{Resultados}

Os resultados da AFE indicaram que a escala 4-IC é fatorável (Teste de esfericidade de Bartlett $=565.9, \mathrm{gl}=6$; $p<.001 ; K M O=.795)$. 0 método de extração de fatores recomendou para uma escala unifatorial como sendo a mais adequada, explicando $81.9 \%$ da variância total (Tabela 2) com valor do coeficiente Ômega de .93. Mais uma vez, a Análise Paralela corroborou a estrutura unidimensional para a escala (Variância dos dados reais, Fator 1: 89.26\%, Fator 2: 10.73\%; Variância dos dados aleatórios, Fator 1: $66.63 \%$, Fator 2: $32.69 \%$ ). Dessa forma, constata-se a semelhança dos resultados obtidos no Estudo 1 e na Subamostra 1 do Estudo 2. Novamente, os índices de ajuste e de resíduo se demonstraram satisfatórios $\chi^{2}=7.83 ; g l=2 ; p<.01 ; R M-$ SEA $[95 \%$ IC $]=.113$ [.000 - .236]; CFI = .997; TLI = 990, com exceção do RMSEA.

Tabela 2. Análise fatorial exploratória da 4-IC $(n=230)$

\begin{tabular}{lc}
\hline \multicolumn{1}{c}{ Item } & Cargas fatoriais \\
\hline $\begin{array}{l}\text { 4. “Eu vejo, eu compro"; esta afirmação } \\
\text { me descreve }\end{array}$ & .90 \\
3. Eu costumo comprar coisas sem pensar & .84 \\
6. "Compro agora e penso sobre isto mais & .78 \\
$\quad$ tarde"; esta afirmação me descreve & \\
2. "Simplesmente Compro"; isto des- & \\
$\quad$ creve a maneira como eu compro as & .77 \\
coisas & \\
Eingenvalue & 3.28 \\
Variância total explicada (\%) & 92.7 \\
Média (DP) & $2.22(1.48)$ \\
Coeficiente Ômega & .93 \\
\hline
\end{tabular}




\section{Subamostra 2}

A segunda amostra foi composta por 362 adultos brasileiros, sendo $71 \%$ mulheres e $29 \%$ homens, a média de idade foi de 24.8 anos ( $D P=12.7), 87.1 \%$ possuíam, pelo menos, o ensino superior completo, $8.3 \%$ possuíam ensino médio completo e $4.7 \%$ ensino fundamental completo. Os respondentes foram, majoritariamente, da região Sudeste do Brasil (51.9\%), seguido do Nordeste (20.6\%), Sul (16.8\%), Centro-Oeste (7.4\%) e Norte (3.3\%).

\section{Instrumentos}

Escala de compra por impulso reduzida de 4 itens (4-CI).

\section{Procedimentos}

De coleta. Para a coleta de dados, foram empregadas as mesmas técnicas de coleta mencionadas no Estudo 1.

De análise. A análise fatorial confirmatória (AFC) foi conduzida a fim de verificar os índices de ajuste dos modelos aos dados. 0 método de extração de máxima semelhança foi utilizado na AFC.

\section{Resultados}

A escala de compra por impulso (4- $\mathrm{Cl} ; n=362)$ apresentou índices de ajuste e de resíduos satisfatórios, $\chi^{2}(2)=3.535$, $p=.171, \chi^{2} / g l=1.77, \mathrm{GFI}=.995, \mathrm{CFI}=.998, \mathrm{TLI}=.994$, RMSEA $=.046$. A confiabilidade composta obteve o valor de .88 , e todos os pesos fatoriais $(\lambda)$ foram estatisticamente significativos e maiores que .75 (Figura 1).

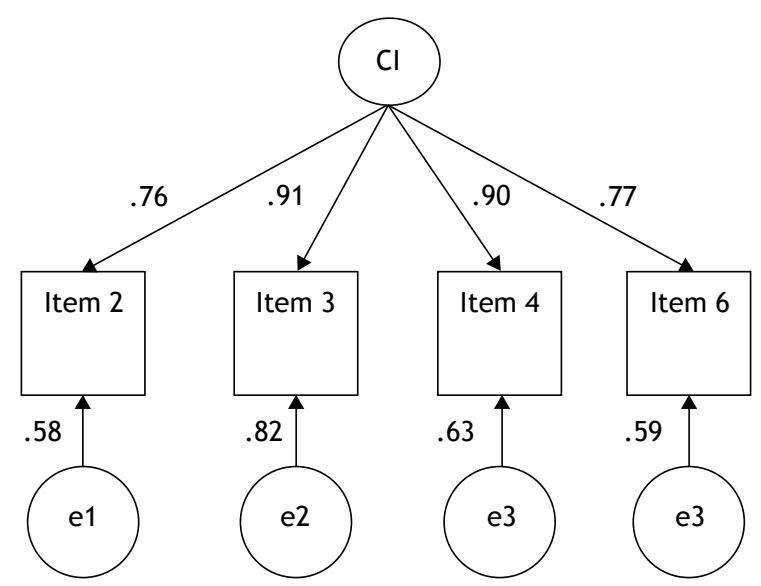

Figura 1. Análise fatorial confirmatória da $4-\mathrm{Cl}$ e pesos fatoriais dos itens.

\section{Estudo 3}

O Estudo 3 teve como objetivo buscar evidências de validade preditiva para a 4-Cl e verificar a invariância do instrumento entre homens e mulheres.

\section{Método}

\section{Participantes}

A amostra foi composta por 1173 adultos brasileiros, sendo $74 \%$ mulheres e $26 \%$ homens, a média de idade foi de
24.8 anos $(D P=12.7), 87 \%$ possuíam, pelo menos, o ensino superior completo, 9.5\% possuíam Ensino Médio completo e 3.2\% Ensino Fundamental completo. Os respondentes foram, majoritariamente, da região Sudeste do Brasil (51.3\%), seguido do Nordeste (19.5\%), Sul (17.3\%), Centro-Oeste (8.3\%) e Norte (3.6\%).

\section{Instrumentos}

Para além da escala de compra por impulso $(4-\mathrm{Cl})$, foram utilizados os seguintes instrumentos.

Materialismo (Richins, 2004). A escala de materialismo é uma escala unidimensional utilizada para aferir a importância dada à posse e aquisição de bens materiais. A escala possui nove itens (e.g., "Eu admiro pessoas que possuem casas, carros e roupas caras"; "Eu gosto de gastar dinheiro com coisas caras"; "Eu gosto de possuir coisas que impressionam as pessoas") e é respondida em uma escala Likert de sete pontos $(1=$ discordo totalmente; $7=$ concordo totalmente, $\alpha=.87)$. Para este estudo foi utilizada a versão brasileira da escala adaptada por Ponchio et al. (2007).

Consumo por Status (Eastman et al., 1999), adaptada para o contexto brasileiro por Strehlau e Aranha (2004). A escala de consumo por status avalia a tendência a compra de bens materiais e serviços visando o status e o prestígio conferidos pela compra. É um instrumento unidimensional composto por três itens (e.g., "Eu me interesso por novos produtos que dão status"; "Eu pagaria mais por produtos de mais status"; "Eu compraria um produto somente porque ele me dá status") respondidos em uma escala Likert de sete pontos $(1=$ discordo totalmente; 7 = concordo totalmente, $\alpha=.89$ ).

Propensão ao endividamento. Esta escala avalia a tendência que uma pessoa tem de endividar-se. 0 instrumento é composto por três itens retirados de escalas que avaliam a propensão ao endividamento. Os itens "Prefiro comprar parcelado a esperar ter dinheiro para comprar à vista" e "Prefiro pagar parcelado mesmo que no total seja mais caro" foram extraídos do estudo de Gathergood e Disney (2011), e o item "Acho normal as pessoas ficarem endividadas para pagar suas coisas" da pesquisa feita por Lea et al. (1995).

\section{Procedimentos}

De coleta. Para a coleta de dados, foram empregadas as mesmas técnicas de coleta mencionadas no Estudo 1.

De análise. Foram realizadas uma análise fatorial confirmatória no Software Mplus (Muthén \& Muthén, 2009), correlações de Person da 4-Cl com as escalas de materialismo, propensão ao endividamento, consumo de status e com a escala original de compra por impulso de nove itens no Software IBM SPSS Statistics (Versão 20), uma análise fatorial confirmatória Multigrupo também no Software Mplus (Muthén \& Muthén, 2009) e Teste $Z$ de Fisher.

\section{Resultados}

A escala de compra por impulso (4- $\mathrm{Cl} ; N=1173)$ apresentou índices de ajuste muito bons: $\chi^{2}(2)=14.436, p=.001$, $\chi^{2} / g l=7.22, \mathrm{GFI}=.994, \mathrm{CFI}=.995, \mathrm{TLI}=.986, \mathrm{RMSEA}=.073$. A confiabilidade composta obteve o valor de .89, e todos os pesos fatoriais $(\lambda)$ foram estatisticamente significativos e maiores que .75 (Figura 2). 


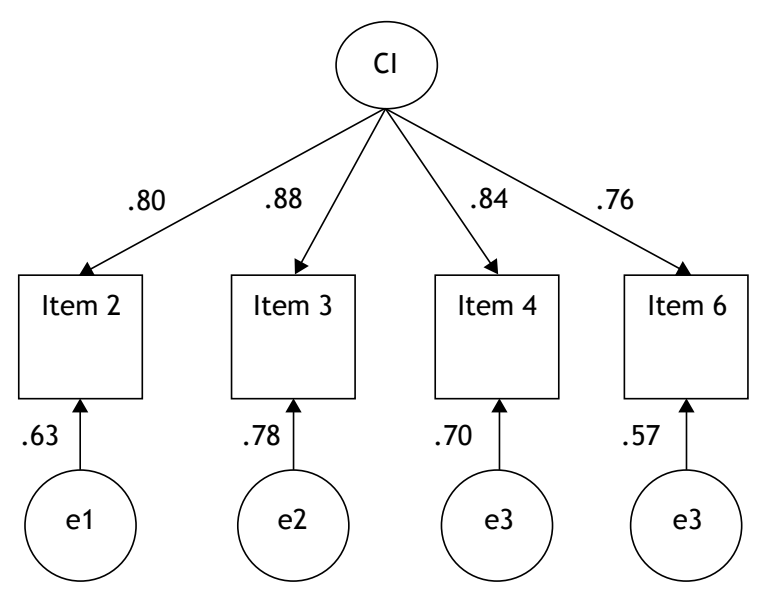

Figura 2. Análise fatorial confirmatória e pesos fatoriais dos itens da $4-\mathrm{Cl}$.

A Tabela 3 apresenta as médias, desvios-padrão e correlação de todas as escalas utilizadas. 0 teste de diferença de correlação $Z$ de Fisher permitiu identificar que as duas escalas de compra por impulso não possuem correlações significativamente diferentes com a escala de materialismo Teste $Z$ de Fisher, $z=-1.163 ; p=0.122, q=.048$, consumo por status Teste $Z$ de Fisher, $z=-0.761 ; p=0.223, q=.031$ e propensão ao endividamento Teste $Z$ de Fisher, $z=-1.175$; $p=0.12, q=.049$. Isso indica que a escala reduzida $(4-\mathrm{Cl})$ mantém suas propriedades de mensuração similares à escala original sem perdas substanciais de informação.

Também foi realizada uma análise fatorial confirmatória multigrupo com o objetivo de investigar em que medida a 4- $\mathrm{Cl}$ era uma medida equivalente para homens e mulheres. Foram testados cinco modelos. A invariância configural avalia em que medida a estrutura do instrumento pode ser considerada equivalente para os diferentes grupos. A invariância métrica investiga se as cargas fatoriais dos itens apresentaram a mesma importância para ambos os sexos. A invariância escalar, por sua vez, investiga se o intercepto dos itens são também equivalentes para ambos os grupos. A invariância da variância fatorial questiona se o traço latente explica a mesma quantidade de variância dos itens. Por fim, a invariância residual investiga se o erro de medida é equivalente entre os grupos.

Para fins de avaliação da invariância do instrumento, foi utilizado o teste de diferença do Comparative Fit Index $(\triangle \mathrm{CFI})$. Para se assumir invariância de medida, o modelo testado não deve apresentar piora nos índices de ajuste superior a .01 no CFI (Cheung \& Rensvold, 2002). Além disso, levando em consideração as conclusões de Fan e Sivo (2009), além de olhar os indicadores isolados, devem ser avaliados os índices de ajuste do modelo como um todo, após a imposição de restrição de equivalência dos parâmetros para os diferentes grupos. Índices de adequação de ajuste adequados indicam aceitabilidade da estrutura proposta (ver critérios sugeridos por Brown, 2006, acima mencionados).

Conforme pode ser visto na Tabela 4, a 4-Cl apresenta invariância de medida para todos os modelos testados, de modo que a medida é considerada completamente equivalente para homens e mulheres.

\section{Considerações finais}

O presente estudo teve como objetivo a redução da escala de compra por impulso de Padilha et al. (2015). As análises realizadas no primeiro e no segundo estudo permitiram corroborar o modelo unifatorial da escala. Além disso, os índices de ajuste da escala reduzida se demonstraram adequados, bem como os índices de consistência interna. 0 terceiro estudo permitiu avaliar que a escala reduzida possuiu correlações de mesma força da escala original. 0 último estudo mostrou que a escala reduzida é invariante entre homens e mulheres, ou seja, quaisquer diferenças entre sexo que sejam identificadas se referem aos diferentes níveis de traço latente, e não uma a uma tendência de enviesamento do instrumento. Por fim, foram encontradas evidências de validade baseada na relação com outras variáveis, visto que a 4-Cl apresentou correlações significativas com a escala de materialismo, consumo por status e propensão ao endividamento.

Entende-se, portanto, que a escala reduzida produzida nesse estudo é um instrumento adequado quando se trata de aferir a compra por impulso. Assim, mais um instrumento encontra-se disponível, no Brasil, para estudos científicos de compra por impulso. Dos benefícios da escala reduzida, destacam-se que elas possuem menor custo de tempo, permitem a inclusão de mais escalas e, por possuir uma estrutura simples, facilita sua utilização em estudos transculturais. Recomenda-se a utilização dessa escala em estudos futuros para averiguar suas qualidades psicométricas em diferentes contextos.

As limitações da pesquisa se dão em algumas instâncias. A primeira delas é a amostra altamente escolarizada e majoritariamente do Sudeste. Apesar da amostra ser relativamente grande, ela não se demonstrou representativa da população brasileira. Assim, estudos futuros que utilizem a escala refinada podem verificar se as propriedades psicométricas apresentadas nesse artigo se mantêm similares em novas amostras. Outra limitação do estudo se refere à pequena quantidade das variáveis relacionadas à compra por impulso que foram utilizadas. Estudos que avaliem as

Tabela 3. Tabela de correlação de Pearson entre compra por impulso 4-IC, (9 itens), materialismo, consumo por status e propensão ao endividamento $(N=1173)$

\begin{tabular}{|c|c|c|c|c|c|c|c|}
\hline Escalas & $M(D P)$ & $\alpha$ & 1 & 2 & 3 & 4 & 5 \\
\hline 1. Compra por impulso (4-IC) & $2.28(1.48)$ & .89 & - & & & & \\
\hline 2. Compra por impulso (9 itens) & $2.95(1.27)$ & .77 & $.92^{* *}$ & - & & & \\
\hline 3. Materialismo & $2.91(1.29)$ & .87 & $.39^{* *}$ & $.43^{* *}$ & - & & \\
\hline 4. Consumo por status & $1.78(1.31)$ & .88 & $.20^{* *}$ & $.23^{* *}$ & $.59^{* *}$ & - & \\
\hline 5. Propensão ao endividamento & $2.38(1.41)$ & .70 & $.40^{* *}$ & $.44^{* *}$ & $.28^{* *}$ & $.16^{* *}$ & - \\
\hline
\end{tabular}

Nota: ${ }^{* *} p<.00$. 
Tabela 4. Índices de ajuste da análise fatorial confirmatória multigrupo (sexo) para a 4-Cl $(N=1173)$

\begin{tabular}{|c|c|c|c|c|c|}
\hline \multirow[t]{2}{*}{ Invariância de medida (sexo) } & \multicolumn{5}{|c|}{ Índices de ajuste } \\
\hline & $\chi^{2} / g l$ & RMSEA $(90 \%$ IC) & SRMR & CFI & $T L I$ \\
\hline Invariância configural & 3.83 & $.049(.025-.076)$ & .02 & .996 & .987 \\
\hline Invariância métrica & 3.15 & $.046(.024-.064)$ & .02 & .994 & .990 \\
\hline Invariância escalar & 3.48 & $.046(.031-.062)$ & .02 & .990 & .989 \\
\hline Invariância da variância fatorial & 4.37 & $.054(.039-.069)$ & .06 & .985 & .985 \\
\hline Invariância residual & 4.31 & $.053(.041-.066)$ & .04 & .980 & .985 \\
\hline
\end{tabular}

$\chi^{2} / g l=$ Chi-quadrado/graus de liberdade, RMSEA = Root Mean Square Error of Approximation, CFI = Comparative Fit Index, TLI = TuckerLewis Index

relações da $4-\mathrm{Cl}$ com variáveis como compra compulsiva e prazer nas compras poderão trazer mais evidências de validade para a escala. Por fim, o presente estudo não realizou análises de teoria de resposta ao item. Novos estudos que utilizem a presente escala poderão avaliar os critérios de dificuldade e discriminação dos itens para que o instrumento seja avaliado por meio de outras análises.

\section{Agradecimentos}

Agradecemos profundamente ao Professor Doutor Bruno Damásio pelo seu apoio na análise de dados e pela parceria na busca da realização de um ótimo trabalho.

\section{Referências}

Ahmad, M. B., Ali, H. F., Malik, M. S., Humayun, A. A., \& Ahmad, S. (2019). Factors affecting impulsive buying behavior with mediating role of positive mood: An empirical study. European Online Journal of Natural and Social Sciences, 8(1), 17-35.

Ahn, J., Lee, S. L., \& Kwon, J. (2020). Impulsive buying in hospitality and tourism journals. Annals of Tourism Research, 102764. https://doi.org/10.1016/j.annals.2019.102764

Aquino, S. D., Natividade, J. C., \& Lins, S. L. B. (2020). Validity evidences of the buying impulsiveness scale in the Brazilian context. Psico-USF, 25(1), 15-25. https://doi.org/10.1590/ 1413-82712020250102

Asparouhov, T., \& Muthén, B. (2010). Weighted least squares estimation with missing data. Mplus Technical Appendix, 2010, 1-10.

Beatty, S. E., \& Ferrell, M. E. (1998). Impulse buying: Modeling its precursors. Journal of Retailing, 74(2), 169-191. https://doi.org/ 10.1016/S0022-4359(99)80092-X

Brown, T. A. (2006). Confirmatory factor analysis for applied researchers. The Guilford Press.

Cacioppo, J. T., \& Petty, R. E. (1982). The need for cognition. Journal of Personality and Social Psychology, 42, 116-131. https:// doi.org/10.1037/0022-3514.42.1.116

Cakanlar, A., \& Nguyen, T. (2019). The influence of culture on impulse buying. Journal of Consumer Marketing, 36(1), 12-23. https://doi.org/10.1108/JCM-03-2017-2139

Cheung, G. W., \& Rensvold, R. B. (2002). Evaluating goodnessof-fit indexes for testing measurement invariance. Structural Equation Modeling, 9(2), 233-255. https://doi.org/10.1207/ S15328007SEM0902_5

Chung, E., \& Fischer, E. (2001). When conspicuous consumption becomes inconspicuous: The case of the migrant Hong Kong consumers. Journal of Consumer Marketing, 18(6), 474-487. https://doi.org/10.1108/07363760110404378
Cook, S. C., \& Yurchisin, J. (2017). Fast fashion environments: consumer's heaven or retailer's nightmare? International Journal of Retail \& Distribution Management, 45(2), 143-157. https:// doi.org/10.1108/ijrdm-03-2016-0027

Dias, S. E. F., Dos Santos, R. M., Martins, V., \& Isabella, G. (2014). Efeitos das estratégias de marketing de compras coletivas sobre o comportamento impulsivo. Revista Brasileira de Marketing, 13(3), 138-151.

Eastman, J., lyer, R., \& Thomas, S. P. (2013). The impact of status consumption on shopping styles: An exploratory look at the millennial generation. Marketing Management Journal, 23(1), 57-73.

Eastman, J. K., Goldsmith, R. E., \& Flynn, L. R. (1999). Status consumption in consumer behavior: Scale development and validation. Journal of Marketing Theory and Practice, 7(3), 41-52. https://doi.org/10.1080/10696679.1999.11501839

Efendi, R., Indartono, S., \& Sukidjo, S. (2019). The mediation of economic literacy on the effect of self control on impulsive buying behaviour moderated by peers. International Journal of Economics and Financial Issues, 9(3), 98-104. https://doi. org/10.32479/ijefi.7738

Fan, X., \& Sivo, S. A. (2009). Using Jgoodness-of-fit indexes in assessing mean structure invariance. Structural equation modeling: A Multidisciplinary Journal, 16(1), 54-69. https://doi. org/10.1080/10705510802561311

Farah, M. F., \& Ramadan, Z. B. (2020). Viability of Amazon's driven innovations targeting shoppers' impulsiveness. Journal of Retailing and Consumer Services, 53, 101973. https://doi.org/ 10.1016/j.jretconser.2019.101973

Fenton-O'Creevy, M., Dibb, S., \& Furnham, A. (2018). Antecedents and consequences of chronic impulsive buying: Can impulsive buying be understood as dysfunctional self-regulation?. Psychology \& Marketing, 35(3), 175-188. https://doi.org/10.1002/ mar.21078

Ferrando, P. J., \& Lorenzo-Seva, U. (2017). Program FACTOR at 10: Origins, development and future directions. Psicothema, 29(2), 236-240.

Gathergood, J., \& Disney, R. (2011). Financial literacy and indebtedness: New evidence for UK consumers. The University of Nottingham. https://doi.org/10.2139/ssrn.1851343

Gosling, S. D., Rentfrow, P. J., \& Swann Jr, W. B. (2003). A very brief measure of the Big-Five personality domains. Journal of Research in Personality, 37(6), 504-528. https://doi.org/10.1016/ S0092-6566(03)00046-1

de Holanda Coelho, G. L., Hanel, P. H., \& Wolf, L. J. (2018). The very efficient assessment of need for cognition: Developing a six-item version. Assessment, 27(8), 1870-1885. https://doi. org/10.1177/1073191118793208

Horn, J. L. (1965). A rationale and technique for estimating the number of factors in factor analysis. Psychometrika, 30(1), 179-185. 
Lea, S. E., Webley, P., \& Walker, C. M. (1995). Psychological factors in consumer debt: Money management, economic socialization, and credit use. Journal of Economic Psychology, 16(4), 681-701. https://doi.org/10.1016/0167-4870(95)00013-4

Lim, X. J., Cheah, J. H., Cham, T. H., Ting, H., \& Memon, M. A. (2020). Compulsive buying of branded apparel, its antecedents, and the mediating role of brand attachment. Asia Pacific Journal of Marketing and Logistics, 32(7), 1539-1563. https://doi.org/10.1108/APJML-03-2019-0126

Lorenzo-Seva, U., \& Ferrando, P. J. (2013). Factor 9.2: A comprehensive program for fitting exploratory and semiconfirmatory factor analysis and IRT models. Applied Psychological Measurement, 37(6), 497-498. https://doi.org/10.1177/0146621613487794

McDonald, R. P. (1999). Test theory: A unified treatment. Lawrence Erlbaum.

Mette, F. M. B., de Matos, C. A., Rohden, S. F., \& Ponchio, M. C. (2019). Explanatory mechanisms of the decision to buy on credit: The role of materialism, impulsivity and financial knowledge. Journal of Behavioral and Experimental Finance, 21, 15-21. https://doi.org/10.1016/j.jbef.2018.10.002

Muthén, L. K., \& Muthén, B. O. (2009). Mplus. Statistical analysis with latent variables. User-s guide, 7. New York: Wiley.

Omar, N. A., Rahim, R. A., Wel, C. A. C., \& Alam, S. S. (2014). Compulsive buying and credit card misuse among credit card holders: The roles of self-esteem, materialism, impulsive buying and budget constraint. Intangible Capital, 10(1), 52-74.

Padilha, M. A., Lins, S., \& Feres-Carneiro, T. (2015). Adaptação da escala de compra por impulso de Rook e Fisher para o contexto brasileiro. Em 45a Reunião Anual da Sociedade Brasileira de Psicologia, Belo Horizonte, MG, Brasil. https://doi. org/10.13140/RG.2.1.3266.7607

Podoshen J. S., \& Andrzejewski S. A. (2012). An examination of the relationships between materialism, conspicuous consumption, impulse buying, brand loyalty. Journal of Marketing Theory and Practice, 20(3), 319-334. https://doi.org/10.2753/ MTP1069-6679200306

Ponchio, M., Aranha, F., \& Todd, S. (2007). Estudo exploratório do construto de materialismo no contexto de consumidores de baixa renda do município de São Paulo. Revistas Gerenciais, 6(1), 13-21.

Puri, R. (1996). Measuring and modifying consumer impulsiveness: A cost-benefit accessibility framework. Journal of Consumer Psychology, 5(2), 87-113. https://doi.org/10.1207/ s15327663jcp0502_01

Richins, M. L. (2004). The Material Values Scale: Measurement properties and development of a short form. Journal of Consumer Research, 31(1), 209-219. https://doi.org/10.1086/383436

Richins, M. L., \& Dawson, S. (1992). A consumer values orientation for materialism and its measurement: Scale development and validation. Journal of Consumer Research, 19(3), 303-316. https://doi.org/10.1086/209304

Rook D. W. (1987). The buying impulse. Journal of Consumer Research, 14(2), 189-199. https://doi.org/10.1086/209105

Rook, D., \& Fisher, R. (1995). Normative influences on impulsive buying behavior. Journal of Consumer Research, 22(3), 305313. https://doi.org/10.1086/209452
Santini, F. O., Lübeck, R. M., \& Sampaio, C. H. (2014). Promoção de desconto: Seus efeitos na compra por impulso e nas intenções de recompra. Gestão \& Planejamento-G\&P, 15(3), 501-520.

Santos, C., \& Fernandes, D. (2006). Avaliação da escala consumer style inventory (CSI) no contexto brasileiro. RAM - Revista de Administração Mackenzie, 7(2), 11-38. https://doi.org/ 10.1590/1678-69712006/administracao.v7n2p11-38

Sharma, P., Sivakumaran, B., \& Marshall, R. (2011). Deliberate self-indulgence versus involuntary loss of self-control: Toward a robust cross-cultural consumer impulsiveness scale. Journal of International Consumer Marketing, 23(3-4), 229-245.

Spiteri Cornish, L. (2020). Why did I buy this? Consumers' post-impulse-consumption experience and its impact on the propensity for future impulse buying behaviour. Journal of Consumer Behaviour, 19(1), 36-46. https://doi.org/10.1002/cb.1792

Stern, H. (1962). The significance of impulse buying today. Journal of Marketing, 26(2), 59-62.

Strehlau, S., \& Aranha, F. (2004). Adaptação e validação da escala de consumo de status (Scs) para uso no contexto brasileiro. Revista de Administração. FACES Journal, 3(1), 9-17.

Tatzel, M. (2002). "Money worlds" and well-being: An integration of money dispositions, materialism and price-related behavior. Journal of Economic Psychology, 23(1), 103-126. https://doi. org/10.1016/S0167-4870(01)00069-1

Thompson, E. R. (2007). Development and validation of an internationally reliable short-form of the Positive and Negative Affect Schedule (PANAS). Journal of Cross-Cultural Psychology, 38(2), 227-242. https://doi.org/10.1177/0022022106297301

Timmerman, M. E., \& Lorenzo-Seva, U. (2011). Dimensionality Assessment of Ordered Polytomous Items with Parallel Analysis. Psychological Methods, 16, 209-220. https://doi.org/10.1037/ a0023353

Verplanken, B., \& Herabadi, A. (2001). Individual differences in impulse buying tendency: Feeling and no thinking. European Journal of Personality, 15(S1), S71-S83. https://doi. org/10.1002/per.423

Vieira, K. M., Ceretta, P. S., Melz, L. J., \& Gastardelo, T. A. R. (2014). Significados do dinheiro e propensão ao endividamento entre alunos universitários. Revista da Faculdade de Administração e Economia, 5(2), 76-103. https://doi.org/10.15603/21769583/refae.v5n2p76-103

Watson, J. J. (2003). The relationship of materialism to spending tendencies, saving, and debt. Journal of Economic Psychology, 24(6), 723-739. https://doi.org/10.1016/j.joep.2003.06.001

Xiao, S. H., \& Nicholson, M. (2011). Mapping impulse buying: A behaviour analysis framework for services marketing and consumer research. The Service Industries Journal, 31(15), 25152528. https://doi.org/10.1080/02642069.2011.531123

Youn, S., \& Faber, R. J. (2002). The dimensional structure of consumer buying impulsivity: Measurement and validation. Advances in Consumer Research, 29, 280. 